\title{
¿Podemos comenzar a cambiar nuestra "mirada" ante la urgencia hipertensiva?
}

Can we start changing our viewpoint towards hypertensive urgency?

Karin Kopitowski , Mercedes Mutchinickł y Esteban Rubinstein

\begin{abstract}
Resumen
La urgencia hipertensiva (UH) es una situación muy frecuente que asusta tanto a los pacientes como a sus familiares y al personal de salud y representa aproximadamente el $30 \%$ de las consultas no programadas (o de urgencia). En el presente artículo, los autores se hacen eco de publicaciones recientes y proponen dejar de utilizar el término urgencia hipertensiva para describir las situaciones de elevación de la presión arterial por encima de una tensión arterial sistólica $\geq 180 \mathrm{mmHg}$ y/o una tensión arterial diastólica (TAD) $\geq 110 \mathrm{mmHg}$, sin asociación con daño de órgano blanco (con o sin sintomatología inespecífica concurrente), ya que los pacientes con este cuadro no corren riesgo inminente, no requieren atención médica de urgencia y, por lo tanto, deberían enmarcarse dentro de los cuidados habituales de los pacientes con factores de riesgo cardiovascular y riesgo cardiovascular global elevado. Esto implica no solo un cambio semántico, sino un desafío en el abordaje y manejo de estas situaciones tan frecuentes.
\end{abstract}

\section{Abstract}

Hypertensive urgency $(\mathrm{UH})$ is a very frequent clinical situation that scares patients, their families as well as health care providers and represents approximately $30 \%$ of the non-scheduled (or urgent) visits. In the present article, taking into account recent publications, the authors propose to stop using the term hypertensive urgency to describe situations of elevated blood pressure above systolic blood pressure $\geq 180 \mathrm{mmHg}$ and / or diastolic blood pressure ( TAD) $\geq 110 \mathrm{mmHg}$, without end organ damage (with or without concurrent non-specific symptomatology), since patients with this condition are not at imminent risk, do not require urgent medical attention and should therefore be framed within usual care of patients with cardiovascular risk factors and high overall cardiovascular risk. This implies not only a semantic change, but a challenge in the approach and management of these frequent situations.

Kopitowski K, Mutchinick M, y Rubinstein E. ¿Podemos comenzar a cambiar nuestra "mirada" ante la urgencia hipertensiva? Evid Act Pract Ambul. 2017;20(4):86-88.

Palabras claves: urgencia hipertensiva, emergencia hipertensiva, hipertensión arterial, manejo. Key words: hypertensive urgency, hypertensive emergency, arterial hypertension, management.

\begin{abstract}
Introducción
La urgencia hipertensiva (UH) es una situación muy frecuente que asusta tanto a los pacientes como a sus familiares y al personal de salud y representa aproximadamente el $30 \%$ de las consultas no programadas (básicamente, en la central de emergencias [CE] $)^{1}$. Suele ocurrir en personas que ya tienen diagnóstico de hipertensión arterial (HTA) y que están o no medicadas, pero también puede presentarse en quienes nunca antes han tenido registros constatados de tensión arterial (TA) elevada. En la mayoría de los casos, los pacientes que consultan por una UH refieren que a partir de un malestar inespecífico, un mareo, una cervicalgia, una cefalea tensional, una situación estresante o un disgusto, se tomaron la TA (en su casa, en una farmacia, etcétera) y, como estaba muy elevada, acudieron (o los mandaron) a la CE asustados ante la presencia de un "pico de presión". También es habitual que algunos individuos con HTA se tomen un día la TA como un "control" y, al constatarla muy elevada, se asusten y acudan o sean enviados "en forma urgente" a una CE con el objetivo de que se les baje rápidamente la TA, asumiendo que, de lo contrario, corren riesgo de sufrir un accidente cerebrovascular (ACV) "inminente".
\end{abstract}

Intentaremos desarrollar este tema reflexionando acerca de la necesidad que expresan muchos autores (y nosotros coincidimos con ellos) de dejar de utilizar el término UH (y "pico de presión") para estas situaciones ya que los pacientes con este cuadro no corren riesgo inminente, no requieren atención médica de urgencia y, por lo tanto, deberían enmarcarse dentro de los cuidados habituales de los pacientes con factores de riesgo cardiovascular (FRCV) y riesgo cardiovascular global (RCG) elevado.

\section{Definición \\ La urgencia hipertensiva (UH) se define como una TA sistóli- ca (TAS) $\geq 180 \mathrm{mmHg}$ y/o una TA diastólica (TAD) $\geq 110$}

$\mathrm{mmHg}$, sin asociación con daño de órgano blanco (DOB). La definición es sencilla si nos fijamos solamente en los valores de la TA, pero se complejiza un poco cuando tenemos que reflexionar acerca de la "asociación o no con DOB". Para facilitar nuestra tarea debemos entender a dicha asociación como una situación "aguda", es decir a la aparición de una "nueva lesión" (DOB nuevo) ocasionada por la TA elevada o a la rápida progresión de un DOB ya conocido (situación también claramente "aguda"). Esto es importante porque si la UH se acompaña de un DOB "agudo" estamos ante un cuadro realmente grave, denominado "emergencia hipertensiva" (EH). El ejemplo más paradigmático de la $\mathrm{EH}$ es la eclampsia, una entidad que todo médico ha estudiado y conoce, en la cual se desencadenan rápidamente mecanismos fisiopatológicos placentarios que ponen en riesgo la vida de la madre y del feto y que la medicina perfectamente "puede salvar" si se actúa pronta y eficazmente. Si reflexionamos acerca de la eclampsia o de otras EH (edema agudo de pulmón hipertensivo, insuficiencia renal aguda hipertensiva, etcétera) podemos ver que en realidad la elevación aguda de la TA es un "signo más" dentro de un síndrome agudo y complejo que pone en riesgo la vida del paciente, pero no es la "causa" del problema. Sin embargo, en todos estos casos, el tratamiento de la HTA forma parte del manejo integral y "urgente" del paciente.

Ahora bien. El concepto más importante que queremos transmitir en este artículo es que la UH no tiene nada que ver con la EH. Otro modo de decir esto es que la UH, en realidad, no es una urgencia. Este último concepto puede resultarle revulsivo a algunos lectores, pero queremos dejar sentada nuestra postura (y la de muchos autores) en el sentido de que la elevación aguda de la TA "sin DOB agudo" (la UH) no debería ser tratada como una urgencia y, por lo tanto, debería "salir" del ámbito de la CE (lo que determinaría que los ámbitos de atención de urgencias y emergencias actuales se libren de atender 
muchísimas consultas) ${ }^{2}$. La idea que deberíamos hacernos cuando pensamos en la $\mathrm{UH}$ es la de un fumador que fuma habitualmente veinte cigarrillos por día y que, durante un día o una semana, está fumando cuarenta. Con este ejemplo podemos ver claramente que no estamos ante una urgencia. Pero la cosa no es tan sencilla ya que ningún fumador acude a una CE porque está fumando más y la gente (y muchos médicos aún) sí considera una urgencia que le suba la presión. Por otra parte, la "emergencia tabáquica" no existe mientras que la EH sí y, aunque se trata de un cuadro muy poco frecuente, es tan "dramático" (en el sentido del riesgo de muerte y la capacidad que tiene la medicina de "salvar la vida") que hace que tanto a los médicos como a los pacientes les cueste mucho separarla de la UH. Esto determina que la conducta a tomar "in situ" ante un paciente con una elevación aguda de la TA no sea tan sencilla. Un aspecto que complejiza también el manejo habitual de la $\mathrm{UH}$ es el tema de los síntomas inespecíficos que muchas veces acompañan a este cuadro (y que no tienen nada que ver con los síntomas relacionados con DOB agudo). Muchos médicos (y pacientes, familiares y farmacéuticos) consideran que si la TA está elevada y el paciente tiene alguno de estos síntomas (cefalea, mareos, cervicalgia, palpitaciones, rubicundez, malestar general, epistaxis, derrame conjuntival, etc.) la situación es "más peligrosa". Esto no es así y es muy importante explicarle a los pacientes (y que los médicos nos convenzamos) que la HTA no es la causa de estos síntomas inespecíficos sino un fenómeno concomitante. Sin embargo, esto forma parte del folklore habitual de lo que ocurre con la UH y es muy difícil salir de la encrucijada del temor que suelen generar estos síntomas. En definitiva, lo que debe quedar claro es que lo que define a la UH es la ausencia de DOB agudo y no la ausencia de síntomas (esto lo veremos mejor cuando hablemos del interrogatorio).

\section{Manejo}

Lo primero que debemos pensar cuando nos consultan por una elevación aguda de la TA es que lo más probable es que se trate de una UH y no de una $\mathrm{EH}$, incluso si el paciente tiene síntomas como cefalea, mareo, cervicalgia, malestar general, etcétera. Esta "mirada" es muy importante ya que si el lector nos acompaña con nuestra postura acerca de que la $\mathrm{UH}$ en realidad no es una urgencia, ya podemos ir pensando que ese paciente no debería ser tratado en la CE (o en cualquier ámbito donde la consulta no es programada) ni tampoco salir de allí con una indicación médica "perentoria". Con el adjetivo "perentorio" queremos decir que tras evaluar a nuestro paciente y determinar que estamos ante una UH no deberíamos indicar ningún tratamiento, ni ninguna medida terapéutica "en ese momento". Esta conducta, obviamente, es controvertida y hablaremos de esto más adelante. Ahora bien. Tener claro que la probabilidad de que un paciente con una elevación aguda de la TA (TAS $\geq$ $180 \mathrm{mmHg}$ y/o una TAD $\geq 110 \mathrm{~mm} \mathrm{Hg}$ ) tenga una $\mathrm{EH}$ es bajísima nos ayuda a aliviar nuestro temor, pero igualmente es preciso descartarla. Esta tarea es relativamente sencilla y podría resumirse de este modo: la $\mathrm{EH}$ es un cuadro clínico en el que el paciente está claramente enfermo, mientras que en la UH el paciente está claramente sano. Esta discriminación se realiza básicamente mediante la anamnesis y el examen físico con el objetivo de evaluar la presencia de DOB agudo. En el interrogatorio es importante preguntar si hubo o hay angor, disnea, náuseas, vómitos, trastornos visuales, dificultad en el habla o la coordinación, déficit motor o sensitivo, confusión, edemas, oliguria y hematuria. En el examen físico deberemos evaluar los signos vitales, realizar un examen neurológico (orientación en tiempo y espacio, presencia de focos motores o sensitivos y rigidez de nuca), un examen cardiovascular (tercer o cuarto ruido, arritmias cardíacas, asimetría en los pulsos periféricos, edemas en los miembros inferiores), un examen respiratorio (estertores crepitantes por insuficiencia cardíaca, signos de edema agudo de pulmón y/o broncoespasmo) y un examen abdominal (soplos aórticos o renales, masas renales o suprarrenales). En la mayoría de los casos con esta información nos alcanza para descartar "DOB agudo" y, por lo tanto, una EH, y no es necesario solicitar estudios complementarios. En este sentido, existe evidencia bibliográfica (ver artículo comentado por Espósito ME, en este número de Evidencia) que demuestra que realizar estudios en búsqueda de $\mathrm{DOB}$ agudo en forma rutinaria en estos pacientes no mostró reducir la morbimortalidad cardiovascular ni a corto ni a mediano plazo) . Esta aseveración, obviamente, no es taxativa y en algunas circunstancias el médico determinará, utilizando el juicio clínico, si es preciso realizar un electrocardiograma (ECG), un fondo de ojo, un laboratorio con uremia, creatininemia, sedimento urinario (para detectar proteinuria, hematuria y cilindros celulares), ionograma en sangre y orina, enzimas cardíacas y una imagen de cerebro en busca de isquemia o hemorragia. Solicitar estudios complementarios utilizando el juicio clínico es un concepto "abierto" y complejo. Hoy en día en muchas CE a estos pacientes se les realiza uno o varios estudios, sin embargo, nuestra propuesta (y la de la literatura que cuestiona el manejo habitual actual de estos pacientes) es la de "bajar un cambio" y volver a confiar principalmente en el interrogatorio y el examen físico para descartar la $\mathrm{EH}$ y evitar los estudios complementarios. El tema evidentemente no es sencillo y seguramente se requieren discusiones en varios niveles para que el médico práctico se sienta respaldado a la hora de tomar estas decisiones.

Ahora bien. Estamos ante la situación (frecuentísima) de estar a cargo de un paciente que nos ha consultado o que ha sido traído a la CE porque se le constató una TAS $\geq 180 \mathrm{~mm} \mathrm{Hg}$ y/o una $T A D \geq 110 \mathrm{~mm} \mathrm{Hg}$ y al que le hemos descartado un "DOB agudo" (idealmente mediante el interrogatorio y el examen físico, pero también puede ser que le hayamos pedido algún estudio, el más habitual un ECG). Este paciente puede estar asintomático o tener síntomas o signos que suelen causar temor (el más común es la cefalea) pero que, como vimos, no son "ni una causa ni una consecuencia" de la elevación de la TA sino síntomas "concomitantes". Estamos entonces, por definición, ante un paciente con una UH. ¿Qué deberíamos hacer, entonces? La respuesta que nos gustaría dar aquí es la siguiente: reasegurarlo en cuanto a que no corre un riesgo inminente, recomendarle que solicite un turno con su médico de cabecera y, eventualmente, tratarle el síntoma "concomitante" que lo aqueja en ese momento (lo más habitual sería indicarle un analgésico para la cefalea tensional).

¿Pero es esto lo que ocurre "en la vida real" con estos pacientes cuando son atendidos en las miles de CE, consultorios de atención no programada y domicilios? Evidentemente no. ¿Por qué no? Porque todavía las "normas" y las guías de práctica clínica indican que la conducta a tomar ante la UH es "bajar la presión" dentro de las primeras 24 a 48 horas. Esto determina que, en la práctica, la mayoría de estos pacientes sean medicados con antihipertensivos "in situ" y sean controlados, también "in situ" o con citas a las 24 o 48 horas, hasta corroborar que la presión haya bajado. Ya hemos manifestado que nosotros (y la literatura que nos gusta y que seguimos) consideramos que esta conducta no tiene sentido y que, además, es incorrecta (aunque no es nuestra intención juzgar a quienes actúan de ese modo). Las razones por las cuales consideramos que es incorrecto tratar a los pacientes con una UH en la CE son dos. Por un lado, la literatura demostró que bajar la presión en forma brusca (sobre todo con drogas endovenosas) en los pacientes que no están cursando una EH puede ocasionar hipotensión severa y posibles 
complicaciones isquémicas (por eso las guías actuales dicen "dentro de las primeras 24 a 48 horas, y por vía oral") ${ }^{4} \mathrm{y}$, por el otro, iniciar un tratamiento antihipertensivo en el ámbito de un consultorio de urgencias puede dar la falsa impresión de que el problema se ha resuelto, que no es necesario un seguimiento clínico a largo plazo y simplifica inapropiadamente el problema. Esta simplificación (en la CE todo parece "perentorio") desvía el manejo de la HTA del paradigma que nosotros proponemos, basado en la valoración del RCG de cada paciente y el vínculo sostenido en el tiempo con el médico de cabecera, que es el más capacitado para tomar decisiones relacionadas con el manejo a largo y mediano plazo de los FRCV ya que es quien más conoce al paciente y a su contexto y quien se encuentra en mejores condiciones para ejercer la toma de decisiones compartidas (TDC). Esta conducta implica no hacer nada con la TA elevada en la CE, acción que muchos colegas confrontan, algunos porque creen que si el paciente no es tratado corre riego de tener un ACV inminente y otros porque consideran que aunque la UH no es ciertamente una urgencia, todo paciente que tiene dos registros de TAS $\geq 160 \mathrm{~mm} \mathrm{Hg}$ y/o TAD $\geq 100 \mathrm{~mm} \mathrm{Hg}$, independientemente del contexto, tiene HTA estadio $2 \mathrm{y}$, por lo tanto, habría que ofrecerle un tratamiento con drogas antihipertensivas. Esta última visión considera que la instancia de la UH es una "oportunidad para tratar" a un individuo que, de otro modo, no consultaría al sistema de salud o bien para aprovechar para ajustarle el tratamiento antihipertensivo a un paciente "mal controlado". Nosotros confrontamos esta creencia por el motivo señalado arriba y, por otra parte, la literatura no logró demostrar que "no dejar escapar" a estos pacientes cuando son atendidos en la CE sirva de algo o disminuya la morbimortalidad'1.

\section{Reflexión final}

La UH representa el $30 \%$ de las consultas en las CE en los países desarrollados. La mayoría de estos pacientes son evaluados en forma relativamente exhaustiva y tratados en forma perentoria en la CE con drogas antihipertensivas para que la presión "baje". Esta conducta se está discutiendo en la literatura y muchos autores consideran que no es correcta. La transición hacia un abordaje racional va a ser lenta. Nuestra postura, manifestada en este artículo, es taxativa en el sentido de que la gran mayoría de los pacientes con una elevación aguda de la TA no tienen una $\mathrm{EH}$ y que esta puede descartarse fácilmente mediante el interrogatorio ( $y$ en menor medida el examen físico) y que los pacientes con una UH (que esperemos prontamente deje de llamarse así) no deberían estudiarse ni tratarse en la CE sino ser referidos a su médico de cabecera para que este aborde la HTA en el marco del manejo de los FRCV, basándose en el RCG individual y utilizando siempre la TDC. Esta postura también implica educar a la población general y al personal de salud acerca del mito del "pico hipertensivo", lo que implica dejar de relacionar a la elevación aguda de la TA con el temor a la muerte o a un evento inminentes, y por lo tanto, "sacar" a estos pacientes de las CE.

\section{Referencias}

1. Basile J, Bloch MJ. Overview of hypertension in adults. Up to Date. 2017 Topic 3852 Version 37.0

2. Heath lona. Hypertensive Urgency-Is This a Useful Diagnosis? JAMA. 2016; 176(7):988-989.

3. Albaladejo Blanco C, y col. Crisis hipertensivas: seudocrisis, urgencias y emergencias. Hipertensión 2014;31:132-42.

4. Esposito ME. El manejo de la urgencia hipertensiva en la central de emergencias no reduce la incidencia de eventos cardiovasculares. Evid Act Pract Ambul. 2017;20(3):xx.. Comentado de: Patel KK, y col. Characteristics and Outcomes of Patients Presenting With Hypertensive Urgency in the Office Setting. JAMA Intern Med. 2016;176(7):981-988. PMID: 27294333.

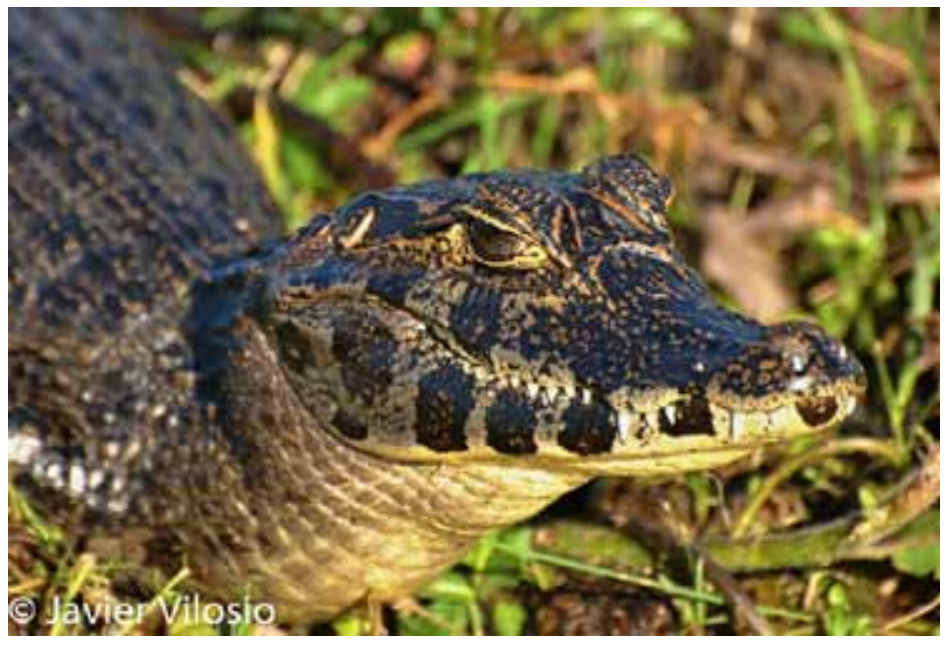

